\title{
Effects of an antibacterial membrane on osteoblast-like cells in vitro
}

This article was published in the following Dove Press journal:

International Journal of Nanomedicine

5 September 201 I

Number of times this article has been viewed

Jun Ye'

Qianqian Yao'

Anchun $\mathrm{Mo}^{2}$

Jing $\mathrm{Nie}^{2}$

Wenwen Liu'

Cui Ye'

Xianji Chen'

'State Key Laboratory of Oral Diseases, ${ }^{2}$ Department of Oral Implant, West China College of Stomatology, Sichuan University,

Chengdu, People's Republic of China

Correspondence: Anchun Mo

No 14, 3rd section, South Renmin Road, Chengdu, 61004I, People's Republic of China

Tel +86288550357 I

Fax +86 2885503630

Email moanchun@I63.com
Abstract: Infection around membranes is often found in guided bone regeneration (GBR). The excellent antibacterial properties of Ag-nHA-nTiO 2 /polyamide-66 (PA66) nanocomposite membranes have been demonstrated previously. The aim of this study was to observe the microstructure of an Ag-nHA-nTiO $/ 2$ PA66 membrane and its effects on osteoblast-like cells in vitro. An Ag-nHA-nTiO $/ 2$ PA66 membrane was used in the experimental group, and both nHA/PA66 and expanded poly tetrafluroethylene (e-PTFE) membranes were set as control. MG63 osteoblast-like cells were cultured on the three kinds of membrane and tissue culture polystyrene (TCP). The microstructure of the above membranes and the cells adhered on them were detected by scanning electronic microscope (SEM). Cell proliferation was determined by 3-(4,5-Dimethylthiazol-2-yl)-2,5-diphenyltetrazolium bromide (MTT) assay, cell viability with a cell viability analyzer, and alkaline phosphatase (ALP) activity and $\mathrm{Ca}^{2+}$ concentration of osteoblast-like cell matrix by enzyme-linked immunosorbent assay. SEM showed that both Ag-nHA-nTiO 2 /PA66 membranes and nHA/PA66 membranes were composed of porous obverse face and smooth opposite face. The e-PTFE membranes showed elliptic surface structure with many tiny lined cracks. The MG63 cells adhered and proliferated well on all three kinds of membranes. Though cell viability on Ag-nHA-nTiO $/ \mathrm{PA}_{6} 6$ membranes was significantly lower than that of the control groups $(P<0.05)$, MTT values, ALP activity, and $\mathrm{Ca}^{2+}$ concentration did not differ significantly among the three kinds of membranes $(P>0.05)$. From these findings, it can be concluded that Ag-nHA-nTiO $/ \mathrm{PA}_{2} 6$ membranes are as biocompatible as nHA/ PA66 membranes and TCP, thus may be applied safely in GBR.

Keywords: guided bone regeneration, hydroxyapatite, polyamide-66, silver, $\mathrm{TiO}_{2,} \mathrm{MG63}$, osteoblast-like cells

\section{Introduction}

The guided bone regeneration (GBR) technique plays an important role in repair of small alveolar bone defects. The principle of GBR is to block the invasion from nonfunctional connective tissue and encourage bone growth with an artificial membrane. ${ }^{1,2}$

The barrier membranes are the most important factor in GBR, because they help maintain a stable environment and secure the priority of osteoblasts growth. ${ }^{3}$ At present, there are many kinds of barrier membranes which generally fall into two categories, namely nonbiodegradable materials and biodegradable materials. Both kinds of membranes risk membrane exposure and subsequent infection, which impair osteogenesis in bone defects. ${ }^{4-6}$ Therefore, the development of antibacterial GBR membranes is important. ${ }^{7-9}$ 
Ag-nHA-nTiO $2 / \mathrm{PA} 66$ nanocomposite membrane is synthesized from Ag-nHA-nTiO ${ }_{2}$ and polyamide, foamed by thermal pressing and injection-molding techniques. ${ }^{11-14}$ The Ag-nHA-nTiO $2 /$ PA66 nanocomposite membrane contains $0.48 \% \mathrm{TiO}_{2}$ and $2.35 \% \mathrm{Ag}^{+}$. $\mathrm{Ag}^{+}$has been used for many years as a broad spectrum antibacterial agent. It has low cytotoxicity on eukaryotic cells, and also has a good antibacterial effect. $\mathrm{Ag}^{+}$is used widely in medicine, including dentistry, ophthalmology, and burn treatment, and in many kinds of biological materials. ${ }^{10}$ Nanometer $\mathrm{TiO}_{2}$ has good antibacterial properties and biological safety, and is nontoxic, tasteless, and non-stimulating. Nanometer $\mathrm{TiO}_{2}$ sterilizes mainly through photocatalysis. Under sunlight, especially ultraviolet light, $\mathrm{TiO}_{2}$ can react with oxygen and water to generate free oxygen and thus achieve an antimicrobial effect. The addition of $\mathrm{Ag}^{+}$produces a synergy effect with $\mathrm{TiO}_{2}$, resulting in an antimicrobial effect under visible light. ${ }^{11}$ Ag-nHA-nTiO $/$ PA66 has been proven to have useful antibacterial properties, especially on Gram-negative bacteria. In our previous study in rats, we found that the osteogenic activity of Ag-nHA-nTiO 2 /PA66 membranes was comparable with that of conventional expanded poly tetrafluroethylene (e-PTFE) membranes and provided a safe strategy for reducing the inflammation response and enhancing bone regeneration, thus promoting the predictability of GBR. ${ }^{12-16}$ However, studies of Ag-nHA-nTiO $/ 2 / \mathrm{PA} 66$ membranes are rare, especially in the area of cytocompatibility. The present study observed the microstructure of Ag-nHA-nTiO $/ 2$ PA66 membranes and the effects of an Ag-nHA-nTiO ${ }_{2} / \mathrm{PA} 66$ nanocomposite membrane on osteoblast-like cells in vitro by scanning electronic microscope (SEM), light microscope, 3-(4,5-Dimethylthiazol2-yl)-2,5-diphenyltetrazolium bromide (MTT) assay, cell viability analyzer (CVA), and enzyme-linked immunosorbent assay (ELISA).

\section{Materials and methods}

\section{Materials and instruments}

The following materials and instruments were used: Ag-nHA-nTiO $2 /$ PA66 membrane (Research Center for Nanobiomaterials of the Sichuan University, Chengdu, China); nHA/PA66 membrane (Research Center for Nano-biomaterials of Sichuan University); e-PTFE membrane (Shanghai Plastic Research Institute, Shanghai, China); titanium circles (Tai Te, Xi An, China); Gibco ${ }^{\circledR}$ F-12 Nutrient Mixture (Invitrogen Corp, Carlsbad, CA) with 10\% fetal bovine serum and penicillin/streptomycin; 6-well, 24-well, and 96-well culture plates (Invitrogen Corp); transferpettor $(50 \mu \mathrm{L}$, $200 \mu \mathrm{L}, 1000 \mu \mathrm{L}$; Gilson International France, Paris, France); paraffin slice machine (Leica RM 215; Leica Microsystems, Wetzlar, Germany); optical microscope (Nikon Eclipse E600; Nikon Instruments Inc, Tokyo, Japan); inverted differential microscope (Olympus IX70; Olympus Corporation, Tokyo, Japan); scanning electronic microscope (JSM-5900; JEOL Ltd, Tokyo, Japan); and a cell viability analyzer (Vi-Cell ${ }^{\circledR}$ Auto, Beckman Coulter, Brea, CA); SPSS Statistics Software (v 13.0; IBM Corp, Armonk, NY).

\section{Cell line}

MG63 osteoblast-like cells (ATCC ${ }^{\mathrm{TM}}$, Manassas, VA). DNA phenotype:Amelogenin:X,Y; CSF1PO:10,12; D13S317:11; D5S818:11,12; D7S820:10; THO1:9,3; TPOX:8,11; $\mathrm{Vw}(\mathrm{A}) 16,19$.

\section{Sample preparation}

Ag-nHA-nTiO 2 /PA66 membrane and nHA/PA66 membrane were white, $1.5 \mathrm{~mm}$ thick. The e-PTFE membrane was white, $1 \mathrm{~mm}$ thick. Biopunches were employed to shape membranes into disk-like forms (14 $\mathrm{mm}$ in diameter) and oblong shapes $(34 \mathrm{~mm} \times 20 \mathrm{~mm})$. The three membranes were rinsed three times with steamed water, loaded into a vitreous dish, and disinfected at high temperature and high pressure.

\section{Methods}

\section{Observation of section structure}

\section{of membranes}

Ag-nHA-nTiO 2 /PA66, nHA/PA66 and e-PTFE membranes were cut into square slices $1 \mathrm{~cm} \times 1 \mathrm{~cm}$, embedded perpendicularly to the section after soaking in wax, made into a paraffin section of $5 \mu \mathrm{m}$ thick, and stained with hematoxylin and eosin stain (H\&E). The cross-sectional structure of membranes was observed with an inverted differential microscope and photographed.

\section{Observation of surface structure of membranes}

Ag-nHA-nTiO 2 /PA66, nHA/PA66 and e-PTFE membranes were cut into square slices $1 \mathrm{~cm} \times 1 \mathrm{~cm}$, dried in vacuum, and coated with gold. The obverse and the reverse of membranes were observed by SEM, and high and low magnification photomicrographs taken.

\section{Cell culture of osteoblastic sarcoma cell MG63}

The freeze-stored tube of MG63 osteoblast-like cells was taken out of liquid nitrogen, placed immediately in a water bath at $37^{\circ} \mathrm{C}$, then centrifuged 3 times $(1000 \mathrm{rpm}$, every 10 minutes). MG63 osteoblast-like cells were maintained in Gibco F-12 Nutrient Mixture with 10\% fetal bovine serum 
and penicillin/streptomycin and seeded in culture bottles at a density of 10,000 cells $/ \mathrm{mL}$. The culture bottles were placed in a $\mathrm{CO}_{2}$ hatch tank $\left(37^{\circ} \mathrm{C}, 5 \% \mathrm{CO}_{2}, 95 \%\right.$ air $)$ and the culture medium was changed every 2-3 days.

\section{Assay of growth curve of MG63 osteoblast-like cells on the three kinds of membrane}

The MG63 osteoblast-like cells were cultured as previously described and seeded at a concentration of 29,000 cells $/ \mathrm{mL}$ in 24-well plates for 1, 3, 5, and 7 days. Cell mixture, $1 \mathrm{~mL}$, was added to each well. Titanium circles ( $14 \mathrm{~mm}$ in diameter) were placed on the membranes. The optical density (OD) of each well was determined at $570 \mathrm{~nm}$ wavelength by MTT assay and the values of six wells in each group were determined each time. Mean OD was calculated for each group and the cell proliferation curve of each group was then drawn.

\section{Assay of cell viability of membranes}

To assess the effects on cell culture viability, the three kinds of membrane and empty wells were seeded with 180,000 cells $/ \mathrm{mL}$ and cultured in six-well plates for 1,3,5, and 7 days. Cell mixture, $2 \mathrm{~mL}$, was added to each well. Cell viability was checked with a CVA. Mean cell viability was calculated for each group and histograms of cell viability were drawn.

\section{Assay of alkaline phosphatase activity and $\mathrm{Ca}^{2+}$ concentration of MG63 osteoblast-like cells on membranes}

The MG63 osteoblast-like cells were cultured as previously described and seeded at a concentration of 220,000 cells $/ \mathrm{mL}$ in six-well plates for 1, 3, 5, and 7 days. Two millilitres of cell mixture was added in each well. Titanium circles (34 mm in diameter) were placed on the membranes. The cells were collected and OD of each hole was determined by ELISA, alkaline phosphatase (ALP) activity and mean $\mathrm{Ca}^{2+}$ values of each group calculated and listed, and figures drawn.

\section{Observation of adhesion and proliferation of the MG63 osteoblast-like cells on the three kinds of membrane}

The MG63 osteoblast-like cells were cultured as previously described and seeded at a concentration of 30,000 cells $/ \mathrm{mL}$ in 24-well plates for 1 and 5 days. Cell mixture, $1 \mathrm{~mL}$, was added in each well. Titanium circles (14 $\mathrm{mm}$ in diameter) were placed on the membranes.

The membranes were rinsed twice in phosphate buffer solution (PBS) liquid (West China, Chengdu, China) after extraction from the plates, fixed in 3\% glutaraldehyde, stored overnight in a refrigerator at $4^{\circ} \mathrm{C}$, gradient dehydrated, soaked in acetic acid isoamyl ester for 20 minutes, dried to a critical point, sprayed with gold, and observed by SEM.

\section{Statistical analysis}

A two-way analysis of variance (ANOVA) test with SPSS software was performed to determine the statistical significance of the differences between values.

\section{Results Observation of section structure of the membranes}

The interconnected pores of various sizes and shapes can be seen in a cross-section of the Ag-nHA-nTiO $/ 2$ PA66 membrane. There were some minor pores within macropores. The structure of the obverse was loose and pore size ranged from 50 to $300 \mu \mathrm{m}$. Macropores of about $300 \mu \mathrm{m}$ can be seen in cross-section from the obverse to the reverse, but the pores cannot be seen in the reverse, which was $50 \mu \mathrm{m}$ thick and very dense (Figure 1A).

The section structure of nHA/PA66 membrane was similar to the Ag-nHA-nTiO $/ 2$ PA66 membrane. The pores in obverse were about $150 \mu \mathrm{m}$ in size and interconnected. Some small pores can be seen in the reverse, which was $100 \mu \mathrm{m}$ thick, and only a handful of pores were $20 \mu \mathrm{m}$ in size, so that the reverse appeared smooth and dense relative to the obverse (Figure 1B).

The section structure of the e-PTFE membrane was uniform. The long cracks, which were $30-50 \mu \mathrm{m}$ in length and interconnected, can be seen from the obverse to the reverse. The obverse and reverse were smooth and their pore size was about $15 \mu \mathrm{m}$ (Figure 1C).

\section{Observation of surface structure of the membranes by SEM}

The interconnected pores of various sizes and shapes can be seen in the obverse surface of the Ag-nHA-nTiO 2 PA66 membrane. Pore size ranged from several microns to 100 microns. Granular sediments can be seen in some pores (Figure 2A). Some small pores $(1-10 \mu \mathrm{m})$ were sparsely localized in the reverse, which was relatively smooth, and most pores were circular. The pores were not interconnected (Figure 2B).

The surface structure of the obverse of the nHA/ PA66 membrane was similar to that of the Ag-nHA-nTiO $/$ PA66 membrane. The pores varied in size and shape and were interconnected. Pores in the surface of the nHA/ PA66 membrane were smaller than those in the surface of 

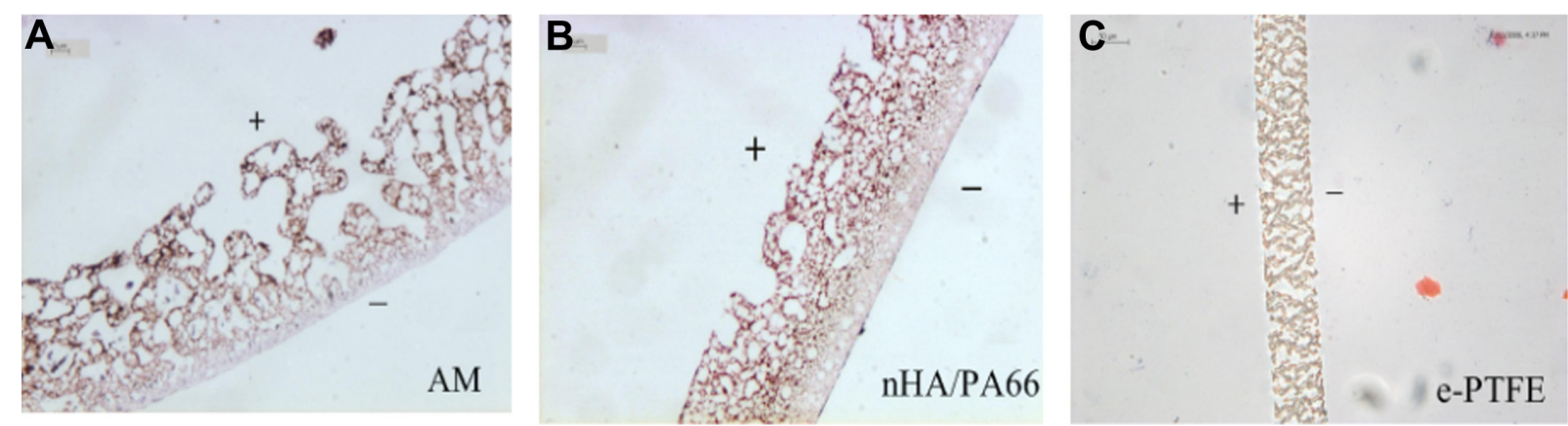

Figure I (A) Microscopic section of the Ag-nHA-nTiO /PA66 (AM) membrane (H\&E $\times$ I00); (B) microscopic section of the nHA/PA66 membrane (H\&E $\times$ I00); (C) microscopic section of the e-PTFE membrane $(\mathrm{H} \& \mathrm{E} \times 200)$.

Notes: + , the observe; - , the reverse.

Abbreviations: e-PTFE, expanded poly tetrafluroethylene; H\&E, hematoxylin and eosin stain; PA66, polyamide-66.

the Ag-nHA-nTiO 2 /PA66 membrane and there were fewer macropores. The surface structure of the reverse of the nHA/ PA66 membrane was similar to that of the Ag-nHA-nTiO $/$ PA66 membrane. Many small pores of about $1 \mu \mathrm{m}$ can be seen, and some sparsely distributed macropores, too (about $10 \mu \mathrm{m}$ ). Most spores were circular, and were not interconnected.

Many long, elliptical, evenly distributed cracks, about $20 \mu \mathrm{m}$ long and $3 \mu \mathrm{m}$ wide, can be seen in the obverse of the e-PTFE membrane. The cracks were ranged neatly and the elongated dense structure can be seen between rows of cracks. The two ends of the cracks were connected to the elongated dense structure. The filamentous structure was on both sides of the cracks. The cracks of upper layer and lower layer were interconnected mutually (Figure 2C). The surface structure of the reverse was the same as that of the obverse (Figure 2D).
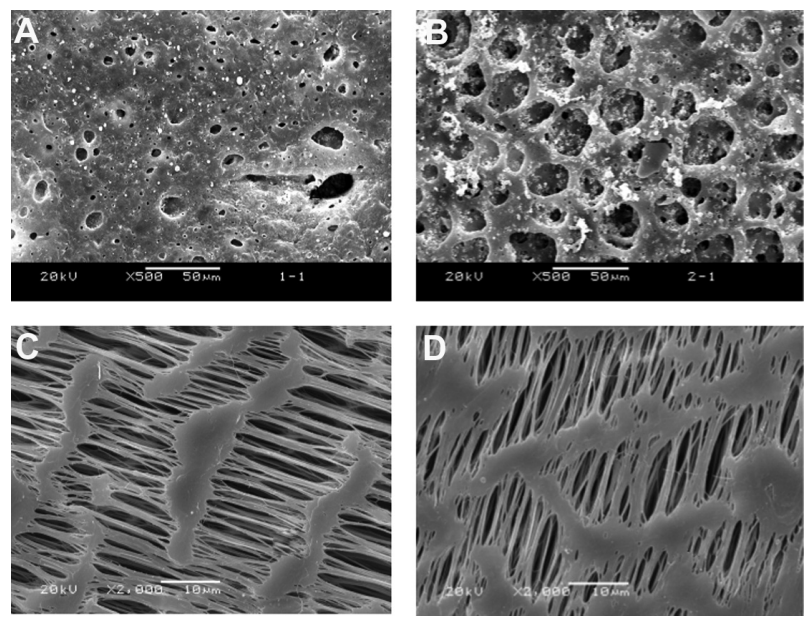

Figure 2 (A) SEM image of surface of obverse of the Ag-nHA-nTiO $/ 2$ PA66 membrane $(\times 500)$; (B) SEM image of surface of reverse of the Ag-nHA-nTiO 2 /PA66 membrane ( $\times 500)$; (C) SEM image of surface of obverse of the e-PTFE membrane $(\times 2000)$; (D) SEM image of surface of reverse of the e-PTFE membrane $(\times 2000)$.

Abbreviations: e-PTFE, expanded poly tetrafluroethylene; PA66, polyamide-66; SEM, scanning electronic microscope.

\section{Microscopic observation of MG63 osteoblast-like cells seeded in the membranes}

Microscopic observation of MG63 osteoblast-like cells seeded in the membranes on the first day showed that cells on the edge of the membranes had fully adhered to the bottom of plates 1 day after seeding. The cells were spindle- and triangle-shaped. It was speculated that the cells had adhered to the membrane surface. Cells in the Ag-nHA-nTiO 2 /PA66, nHA/PA66, and e-PTFE membranes were the same shape.

\section{Assay of growth curve of MG63 osteoblast-like cells in the three kinds of membranes}

MTT values of MG63 osteoblast-like cells seeded in the three kinds of membranes showed clear proliferation trends at 1, 3, 5, and 7 days (Table 1). MTT values at 1, 3, 5, and 7 days of experimental, control, and blank groups differed significantly $(P<0.05)$; values increased from 1 day through to 7 days. MTT values of experimental and control groups did not differ $(P>0.05)$.

\section{Assay of cell viability on the membranes}

Cell viability of the four groups at four time points was about 84\% (Table 2). Cell viability of MG63 osteoblast-like cells on the Ag-nHA-nTiO 2 /PA66 membranes at 5 days was lower at $79.4 \%$ and increased by 7 days. But clear trends in cell viability variation at four time points were not found in the other groups, the cell viability of which ranged from $80 \%$ to 90\%. Cell viability of the Ag-nHA-nTiO $/ 2$ PA66, nHA/PA66, and e-PTFE membranes and blank group did not differ significantly at $1,3,5$, and 7 days $(P>0.05)$. Cell viability differed significantly between the Ag-nHA-nTiO $2 / \mathrm{PA} 66, \mathrm{nHA} /$ PA66 and e-PTFE membranes and blank group $(P<0.05)$. 
Table I MTT values of experimental and control groups at different times

\begin{tabular}{lllll}
\hline Group & I day & 3 day & $\mathbf{5}$ day & $\mathbf{7}$ day \\
\hline Control & $0.3641 \pm 0.0197$ & $0.5541 \pm 0.0594$ & $1.8806 \pm 0.0391$ & $2.1974 \pm 0.1239$ \\
e-PTFE & $0.2192 \pm 0.0110$ & $0.5673 \pm 0.0463$ & $0.8189 \pm 0.0715$ & $1.1510 \pm 0.0541$ \\
nHA/PA66 & $0.2990 \pm 0.0289$ & $0.5614 \pm 0.0205$ & $0.9847 \pm 0.0395$ & $1.5698 \pm 0.0193$ \\
Ag-nHA-nTiO $/$ PA66 & $0.2565 \pm 0.0160$ & $0.4288 \pm 0.0097$ & $0.6949 \pm 0.0062$ & $2.0461 \pm 0.2849$ \\
\hline
\end{tabular}

Notes: Values mean $\pm \mathrm{SD}, \mathrm{n}=3$ (Abs).

Abbreviations: e-PTFE, expanded poly tetrafluroethylene; MTT, 3-(4,5-Dimethylthiazol-2-yl)-2,5-diphenyltetrazolium bromide assay; n, number; PA66, polyamide-66; SD, standard deviation.

Cell viability of the Ag-nHA-nTiO $/ 2 / \mathrm{PA} 66$ membranes was lower than that of other groups but cell viability did not differ significantly between the nHA/PA66 and e-PTFE membranes and blank group $(P>0.05)$.

\section{Assay of alkaline phosphatase activity and $\mathrm{Ca}^{2+}$ concentration of MG63 osteoblast-like cells on the membranes}

ALP activity differed significantly between different time points $(P<0.05)$, but there was no statistically significant difference between days 3 and 5 by a two-way ANOVA method, or between groups $(P>0.05)$ (Table 3$)$.

$\mathrm{Ca}^{2+}$ concentration of MG63 osteoblast-like cells on the Ag-nHA-nTiO $2 /$ PA66, nHA/PA66, and e-PTFE membranes and blank group showed a trend for MG63 osteoblast-like cells to proliferate in general and for them to decline somewhat from days 3 to 5 (Table 4 ). $\mathrm{Ca}^{2+}$ concentration differed significantly between different time points $(P<0.05)$. It was speculated that cell viability differed between different time points. Further paired comparison by ANOVA showed that $\mathrm{Ca}^{2+}$ concentration did not differ significantly between days 3 and $5(P>0.05)$ and $\mathrm{Ca}^{2+}$ concentration at day 7 was the highest. $\mathrm{Ca}^{2+}$ concentration did not differ significantly between the different groups $(P>0.05)$.

\section{Observation of adhesion and proliferation of the MG63 osteoblast-like cells on the three kinds of membrane} Adhesion and proliferation of the MG63 osteoblastlike cells on the Ag-nHA-nTiO $/ 2$ PA66 membrane The adhesion of MG63 osteoblast-like cells on the Ag-nHA$\mathrm{nTiO}_{2} / \mathrm{PA} 66$ membrane on day 1 is shown in Figure 3A and B. Figure 3A shows that the MG63 osteoblast-like cells on the Ag-nHA-nTiO $/$ PA66 membrane had spread, and the pseudopods of the cells adhered to the membrane surface and membrane pores. Figure 3B shows that the cells adhered to the edge and the middle of the pores. Some cells that the cells may have proliferated from can be seen adjacent to several pores, too. Cells were long spindle- and triangle-shaped, and the pseudopods were fully spread. The membrane had good compatibility with and adhesion to the MG63 osteoblast-like cells.

The proliferation of the MG63 osteoblast-like cells on the Ag-nHA-nTiO $/ 2$ PA66 membrane on day 5 is shown in Figure $3 \mathrm{C}$ and D. Figure $3 \mathrm{C}$ shows the interconnection of the cells. Cells were long spindle-shaped, the pseudopods were connected to nearby cells, and some cells that the cells may have proliferated from can be seen adjacent to several pores, too. One part of some cells adhered to the membrane surface and another part stretched deep into the pores, which the cells may have proliferated from the middle of the pores to the surface. Figure 3D shows long spindle- and triangleshaped MG63 osteoblast-like cells on the membrane surface. It can be observed that some cells adhered to the edge of the membrane, and some cells stacked with each other. Some cells that the cells may have proliferated from can be seen adjacent to several pores, too.

In general, the MG63 osteoblast-like cells grew well on the Ag-nHA-nTiO 2 /PA66 membrane. It was speculated that the cells in the pores also grew well because there were more and bigger pores on the obverse of the membrane and the visible cells were distributed mainly on the edge of the pores.

Table 2 Mean CVA values of MG63 osteoblast-like cells on experimental and control groups

\begin{tabular}{lllll}
\hline Group & I day & 3 day & 5 day & 7 day \\
\hline Control & $92.333 \pm 2.9023$ & $87.033 \pm 3.0039$ & $85.967 \pm 1.6773$ & $84.400 \pm 5.6027$ \\
e-PTFE & $92.733 \pm 0.9074$ & $87.100 \pm 3.1433$ & $85.633 \pm 5.3632$ & $84.333 \pm 10.564$ \\
nHA/PA66 & $88.533 \pm 8.2972$ & $85.700 \pm 8.2972$ & $89.567 \pm 2.3459$ & $88.900 \pm 0.7000$ \\
Ag-nHA-nTiO 2 /PA66 & $80.333 \pm 2.4987$ & $82.667 \pm 3.3606$ & $79.400 \pm 2.3388$ & $85.933 \pm 1.2662$ \\
\hline
\end{tabular}

Notes: Values mean $\pm S D, n=3(\%)$.

Abbreviations: CVA, cell viability analyser; e-PTFE, expanded poly tetrafluroethylene; $n$, number; PA66, polyamide-66; SD, standard deviation. 
Table 3 Alkaline phosphatase activity at I, 3, 5, and 7 days in experimental and control groups

\begin{tabular}{lllll}
\hline Group & I day & 3 day & $\mathbf{5}$ day & $\mathbf{7}$ day \\
\hline Control & $0.037 \pm 0.5688$ & $0.591 I \pm 1.425 I$ & $3.5097 \pm 0.8465$ & $8.3313 \pm 1.9420$ \\
e-PTFE & $0.0739 \pm 1.1375$ & $2.3644 \pm 0.7863$ & $1.9211 \pm 0.9295$ & $4.9875 \pm 1.4662$ \\
nHA/PA66 & $0.5012 \pm 0.2813$ & $1.8491 \pm 0.4825$ & $2.4832 \pm 0.5173$ & $5.0572 \pm 0.8714$ \\
Ag-nHA-nTiO 2 PA66 & $1.1453 \pm 0.8392$ & $0.628 I \pm 0.4196$ & $1.4846 \pm 0.5793$ & $5.0879 \pm 0.1123$ \\
\hline
\end{tabular}

Notes: Values mean $\pm \mathrm{SD}(\mathrm{U} / \mathrm{mL}), \mathrm{n}=3$.

Abbreviations: e-PTFE, expanded poly tetrafluroethylene; n, number; PA66, polyamide-66; SD, standard deviation.

\section{Adhesion and proliferation of the MG63} osteoblast-like cells on the nHA/PA66 membrane

The adhesion of the MG63 osteoblast-like cells on the nHA/PA66 membrane on day 1 were the same as on the Ag-nHA-nTiO ${ }_{2}$ PA66 membrane (Figure 4A and B). Figure 4A shows that the MG63 osteoblast-like cells on the nHA/ PA66 membrane had spread, the pseudopods had adhered to the membrane surface and pores. Figure $4 \mathrm{~B}$ shows that the cells adhered to the edge of and the middle of the pores and some cells that the cells may have proliferated from can be seen adjacent to several pores, too. Cells were long spindle-shaped, and the pseudopods fully spread. The membrane had good compatibility with and adhesion to the MG63 osteoblast-like cells.

The proliferation of the MG63 osteoblast-like cells on the nHA/PA66 membrane on day 5 was the same as on the AgnHA-nTiO ${ }_{2} /$ PA66 membrane (Figure 4C and D). Figure 4C shows that the cells were interconnected and long spindleshaped, the pseudopods were connected to nearby cells, and some cells that the cells may have proliferated from can be seen adjacent to several pores, too. One part of some cells adhered to the membrane surface and another part stretched deeply into the pores, which the cells may have proliferated from the middle of the pores to the surface. Figure 4D shows the long spindle- and triangle-shaped MG63 osteoblast-like cells on the membrane surface. It can be observed that some cells adhered to the edge of the membrane, and some cells stacked with each other. Some cells that the cells may have proliferated from can be seen adjacent to several pores, too.

In general, the MG63 osteoblast-like cells grew well on the nHA/PA66 membrane. It was speculated that the cells in the pores also grew well because there were more and bigger pores on the obverse of the membrane and the visible cells were distributed mainly on the edge of the pores.

\section{Adhesion and proliferation of the MG63 osteoblast-like cells on the e-PTFE membrane}

Adhesion of the MG63 osteoblast-like cells on the e-PTFE membrane on day 1 is shown in Figure 5A and B. Figure 5A shows that the pseudopods were insufficiently stretched out, lying on the membrane surface and not stretching into the cracks. Figure 5B shows that a few of the cells scattered on the surface of the membrane. The cells varied in shape, such as long spindle, triangle, and square. The pseudopods did not stretch well. So it was speculated that the adhesion of the MG63 cells on the e-PTFE membrane was common.

The proliferation of the MG63 osteoblast-like cells on the e-PTFE membrane on day 5 is shown in Figure 5C and D. Figure 5C shows that the pseudopods of the cells were interconnected and that many microfilaments were interconnected mutually, too. Figure 5D clearly shows that the cells proliferated to form a dense layer. Cells were mainly spindle- and triangle-shaped, and were interconnected and spread on the membrane surface. In general, the adhesion of the MG63 osteoblast-like cells on the e-PTFE membrane was not very good, possibly because of their hydrophobic property, but the proliferation of the MG63 osteoblast-like cells on the e-PTFE membrane was good enough.

In general, the adhesion and proliferation of the MG63 osteoblast-like cells on the Ag-nHA-nTiO $/ 2 / \mathrm{PA} 66$ and nHA/ PA66 membranes were good and better than on the e-PTFE membrane.

Table $4 \mathrm{Ca}^{2+}$ concentration at I, 3, 5, and 7 days in experimental and control groups

\begin{tabular}{lllll}
\hline Group & I day & $\mathbf{3}$ day & $\mathbf{5}$ day & $\mathbf{7}$ day \\
\hline Control & $0.0378 \pm 0.0071$ & $0.2238 \pm 0.0691$ & $0.1600 \pm 0.0359$ & $0.5931 \pm 0.7825$ \\
e-PTFE & $0.0397 \pm 0.0103$ & $0.1640 \pm 0.0272$ & $0.1295 \pm 0.0421$ & $0.3680 \pm 0.4982$ \\
nHA/PA66 & $0.0355 \pm 0.0153$ & $0.1220 \pm 0.0468$ & $0.1117 \pm 0.0285$ & $0.4477 \pm 0.4227$ \\
Ag-nHA-nTiO_/PA66 & $0.0387 \pm 0.0661$ & $0.1053 \pm 0.0260$ & $0.1288 \pm 0.0252$ & $0.3592 \pm 0.4823$ \\
\hline
\end{tabular}

Notes: Values mean $\pm \mathrm{SD}(\mu \mathrm{mol} / \mathrm{mL}), \mathrm{n}=3$.

Abbreviations: e-PTFE, expanded poly tetrafluroethylene; n, number; PA66, polyamide-66; SD, standard deviation. 

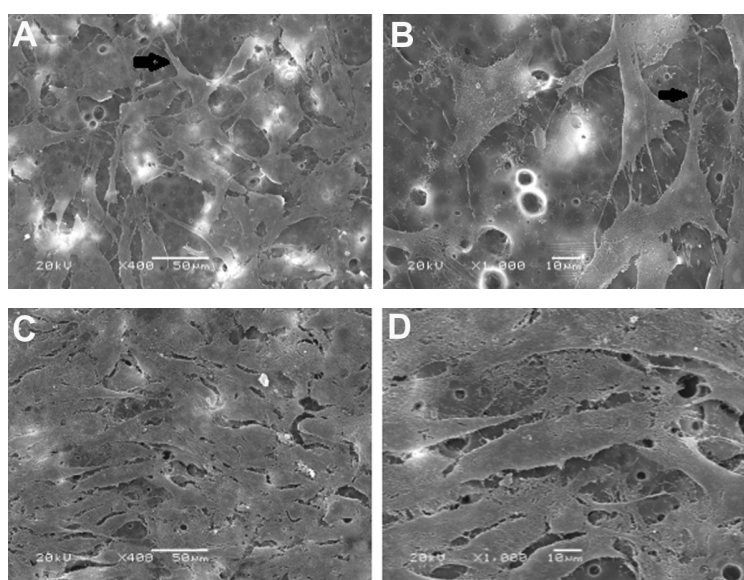

Figure 3 (A and B) Adhesion of MG63 osteoblast-like cells on day I on the Ag-nHA-nTiO $/$ PA66 membrane (SEM * 400)/(SEM * I000). (C and D) Adhesion of MG63 osteoblast-like cells on day 5 day on the Ag-nHA-nTiO $/ 2$ PA66 membrane $(\mathrm{SEM} * 400) /(\mathrm{SEM} * 1000)$.

Abbreviations: PA66, polyamide-66; SEM, scanning electronic microscope.

\section{Discussion}

The Ag-nHA-nTiO $/ 2$ PA66 membrane used was a nonbiodegradable material. It overcame the usual shortcoming of nonbiodegradable membranes of needing a second surgery to be moved, and also had good antibacterial properties and mechanical properties. The tissue compatibility and bone induction activity of the Ag-nHA-nTiO 2 /PA66 membrane have not been studied previously. The present experiment studied effects of the Ag-nHA-nTiO 2 /PA66 membrane on osteoblast-like cells in vitro. The results showed that the $\mathrm{Ag}-\mathrm{nHA}-\mathrm{nTiO}_{2} / \mathrm{PA} 66 \mathrm{mem}-$ brane had no negative effects on growth of osteoblast-like cells, helped adhesion of the MG63 osteoblast-like cells, and, thus, had excellent tissue compatibility.

MTT values indicated a trend for cell proliferation, as measured at $1,3,5$, and 7 days in blank, control,
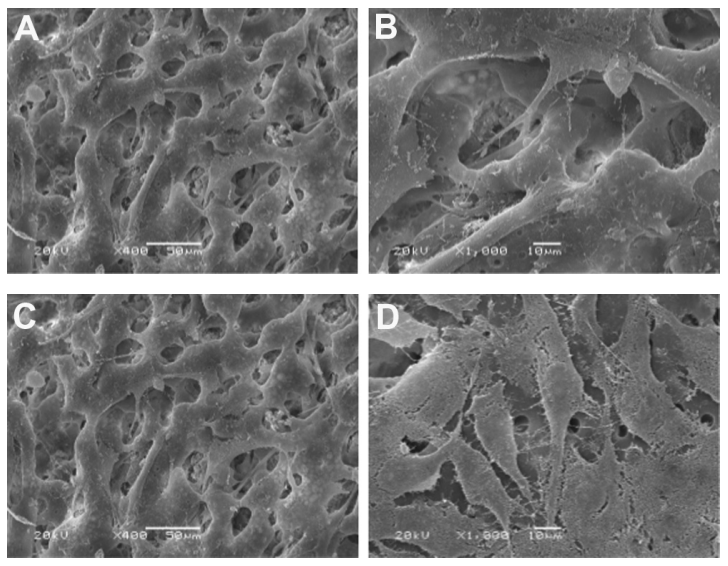

Figure 4 (A and B) Adhesion of MG63 osteoblast-like cells on day I on the nHA/PA66 membrane (SEM * 400)/(SEM * 1000). (C and D) Adhesion of MG63 osteoblast-like cells on day 5 on the nHA/PA66 membrane (SEM* 400)/(SEM* 1000). Abbreviations: PA66, polyamide-66; SEM, scanning electronic microscope.
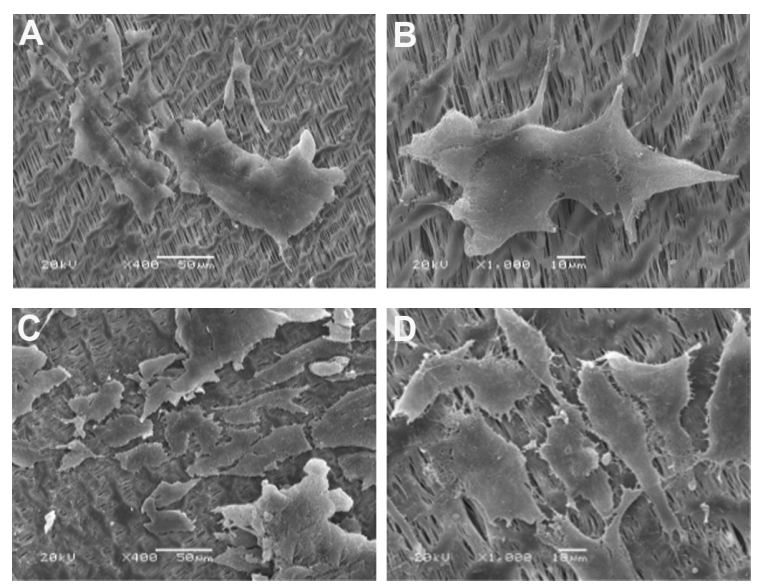

Figure 5 (A and B) Adhesion of MG63 osteoblast-like cells on day I on the e-PTFE membrane (SEM * 400)/(SEM * 1000). (C and D) Attachment of MG63 osteoblastlike cells on day 5 day on the e-PTFE membrane (SEM * 400)/(SEM * 1000). Abbreviations: e-PTFE, expanded poly tetrafluroethylene; SEM, scanning electronic microscope.

and experimental groups, but there were no statistically significant differences between control and experimental groups $(P>0.05)$. Cells grew well on the Ag-nHA-nTiO $/$ PA66 membrane and the membrane had no negative effects on MG63 osteoblastic cell proliferation. Ag-nHA-nTiO $/$ PA66 and nHA/PA66 membranes absorbed the dye during the dissolution of violet crystallization in the two membranes by DMSO, and had been dyed blue at days 5 and 7 especially. Therefore, it was presumed that the measured OD values were smaller than actual values. So this further illustrated that the two membranes had no negative effects on the growth of MG63 osteoblast-like cells, and their growth on the two membranes was better than on the e-PTFE membrane. The reason for absorption of dye by the two membranes may be because of (1) the absorption of dye by nHA or because (2) the surfaces of the two membranes in which cells seeded were loose and porous, the cells grew in the pores, and the violet crystallization was difficult to dissolve out.

The results showed that viability of MG63 osteoblastic cells on the three membranes did not decline over time, showing that MG63 osteoblastic cells can proliferate normally on all three membranes. Cell viability differed significantly between the Ag-nHA-nTiO 2 /PA66 membrane and control groups. Some white particles were precipitated from the Ag-nHA-nTiO $/$ /PA66 membrane during cell collection. These tiny particles were analyzed by CVA together with osteoblastic cells during sampling. We speculated that during analysis these tiny particles were treated as dead cells, and therefore the calculated cell viability value was lower than the actual value, which may explain why cell viability in the experimental group was lower than in the control 
group. Another possibility was the antibiosis of $\mathrm{Ag}^{+}$and $\mathrm{TiO}_{2}$ in the Ag-nHA-nTiO $/ 2$ PA66 membrane. Although the effect of $\mathrm{Ag}^{+}$and $\mathrm{TiO}_{2}$ on eukaryotic cells is very small, a tiny inhibition on cell proliferation can be detected in cell culture in vitro. So we need to detect ALP activity to judge which explanation is correct.

ALP activity reflects the proliferation and osteogenesis activity of MG63 osteoblast cells and was considered an early sign of the differentiation and maturation of osteoblast cells. The experimental results showed that the activity of ALP of MG63 osteoblast-like cells of experimental, control, and blank groups increased over the 7 days. ALP activity between 3 days and 5 days did not differ significantly $(P>0.05)$, which indicated that the osteogenetic activity of MG63 osteoblast-like cells was stable during this time. ALP activity significantly increased between 5 and 7 days, and we can speculate that MG63 osteoblast-like cells changed from proliferation to aggregation and prepared for further calcification. The ALP activity between the experimental, control, and blank groups did not differ significantly, further proving the good biocompatibility of the three kinds of membrane. No inhibition of differentiation and functions of MG63 osteoblast-like cells was found in the Ag-nHA-nTiO $/ 2$ PA66 membrane.

The results for $\mathrm{Ca}^{2+}$ activity were consistent with those for ALP, further proving that there was no negative effect of the Ag-nHA-nTiO $/ 2$ PA66 membrane on growth of MG63 osteoblast-like cells. It was found that the cells cannot be completely digested and collected because of the loose porous structure of the Ag-nHA-nTiO 2 / $\mathrm{PA} 66$ and nHA/ PA66 membrane surface in the experiment to detect MG63 osteoblast-like cell viability. Therefore the calculated value of cell viability was lower than the actual value because the cells were collected only partially. Therefore the experimental results have fully proven that the Ag-nHA-nTiO $/$ PA66 membrane has good biocompatibility with MG63 osteoblast-like cells.

The adhesion and proliferation of MG63 osteoblastlike cells, which were cultured on the Ag-nHA-nTiO $/$ PA66 membrane, were observed at days 1 and 5 in the cell adhesion experiment. The experimental and control groups had good cell compatibility for adhesion and proliferation of MG63 osteoblast-like cells. The porous surface of Ag-nHA-nTiO $/ 2$ PA66 membrane was more beneficial to proliferation and adhesion of MG63 osteoblast-like cells. The Ag-nHA-nTiO 2 /PA66 membrane and nHA/PA66 membrane are bioactive materials, while the e-PTFE membrane is biologically inert. Theoretically, the cell compatibility of osteoblasts of bioactive materials is lower than that of biologically inert materials. The adhesion, spread, and traffic between cells of MG63 osteoblast-like cells in the Ag-nHA-nTiO ${ }_{2} / \mathrm{PA} 66$ membrane and nHA/PA66 membrane are superior to that of that e-PTFE membrane. This result can explain why the Ag-nHA-nTiO $2 / \mathrm{PA} 66$ and nHA/ PA66 membranes, as GBR membrane materials, do not need a second surgery to be removed, while the e-PTFE membrane does.

Because the adhesion and proliferation of MG63 osteoblast-like cells in the Ag-nHA-nTiO 2 / $\mathrm{PA} 66$ membrane group were comparable with those in the nHA/PA66 membrane group and the e-PTFE membrane group, we can conclude that the $\mathrm{Ag}^{+}, \mathrm{TiO}_{2}$, and PA66 ingredients in the experimental membrane were biological.

\section{Conclusion}

The Ag-nHA-nTiO $2 / \mathrm{PA}_{2} 6$ membrane had no negative effects on growth of osteoblast-like cells and the loose porous structure of the membrane helped the adhesion and proliferation of osteoblast-like cells. Because the Ag-nHA-nTiO $/ 2$ PA66 membrane has good biocompatibility and a graded structure, it could be superior to the e-PTFE membrane.

\section{Disclosure}

The authors report no conflicts of interest in this work.

\section{References}

1. Dahlin C, Simion M, Nanmark U, Sennerby L. Histological morphology of the e-PTFE/tissue interface in humans subjected to guided bone regeneration in conjunction with oral implant treatment. Clin Oral Implants Res. 1998;9:100-106.

2. Dupoirieux L, Pourquier D, Picot MC, Neves M. Comparative study of three different membranes for guided bone regeneration of rat cranial defects. Int J Oral Maxillofac Surg. 2001;30(1):58-62.

3. Nyman R, Magnusson M, Sennerby L, Nyman S, Lundgren D. Membrane-guided bone regeneration. Segmental radius defects studied in the rabbit. Acta Orthop Scand. 1995;66(2):169-173.

4. Tempro PJ, Nalbandian J. Colonization of retrieved polytetrafluoroethylene membranes: morphological and microbiological observations. J Periodontol. 1993;64(3):162-168.

5. Rominger JW, Triplett RG. The use of guided tissue regeneration to improve implant osseointegration. J Oral Maxillofac Surg. 1994;52(2):106-112.

6. Engelke WG, Diederichs CG, Jacobs HG, Deckwer I. Alveolar reconstruction with splitting osteotomy and microfixation of implants. Int $J$ Oral Maxillofac Implants. 1997;12(3):310-318.

7. Chen G, Ushida T, Tateishi T. Development of biodegradable porous scaffolds for tissue engineering. Mater Sci Eng C Mater Biol Appl. 2001;17(1-2):63-69.

8. Hailab NJ, Bundy KJ, O`Connor K, Moses RL, Jacobs JJ. Evaluation of metallic and polymeric biomaterial surface energy and surface roughness characteristics for directed cell adhesion. Tissue Eng. 2001;7(1):55-69.

9. Cerroni L, Filocamo R, Fabbri M, Piconi C, Caropreso S, Condò SG. Growth of osteoblast-like cells on porous hydroxyapatite ceramics: an in vitro study. Biomol Eng. 2002;19(2-6):119-124. 
10. Zhang J, Xu Q, Huang C, Mo A, Li J, Zuo Y. Biological properties of an anti-bacterial membrane for guided bone regeneration: an experimental study in rats. Clin Oral Implants Res. 2010;21(3):321-327.

11. Juan L, Zhimin Z, Anchun M, Lei L, Jingchao Z. Deposition of silver nanoparticles on titanium surface for antibacterial effect. Int $J$ Nanomedicine. 2010;5:1-7.

12. Zhang J, Huang C, Xu Q, Mo A, Li J, Zuo Y. Biological properties of a biomimetic membrane for guided tissue regeneration: a study in rat calvarial defects. Clin Oral Implants Res. 2010;21(4):392-397.

13. Mo AC, Xu W, Xian S, Li YB, Bai S. Antibacterial activity of silverhydroxyapatite/titania nanocomposite coating on titanium against oral bacteria. Key Eng Mater. 2007;330:455-458.
14. Xu Q, Lu H, Zhang J, Lu G, Deng Z, Mo A. Tissue engineering scaffold material of porous nanohydroxyapatite/polyamide 66 . Int J Nanomedicine. 2010;5:331-335.

15. Zhang JC, Lu HY, Lv GY, Mo AC, Yan YG, Huang C. The repair of critical-size defects with porous hydroxyapatite/polyamide nanocomposite: an experimental study in rabbit mandibles. Int J Oral Maxillofac Surg. 2010;39(5):469-477.

16. Meng Y, Liu M, Wang SA, et al. Cellular reactions of osteoblast-like cells to a novel nanocomposite membrane for guided bone regeneration Appl Surf Sci. 2008;255(2):267-269.
International Journal of Nanomedicine

\section{Publish your work in this journal}

The International Journal of Nanomedicine is an international, peerreviewed journal focusing on the application of nanotechnology in diagnostics, therapeutics, and drug delivery systems throughout the biomedical field. This journal is indexed on PubMed Central,

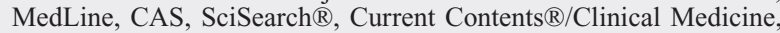

\section{Dovepress}

Journal Citation Reports/Science Edition, EMBase, Scopus and the Elsevier Bibliographic databases. The manuscript management system is completely online and includes a very quick and fair peer-review system, which is all easy to use. Visit http://www.dovepress.com/ testimonials.php to read real quotes from published authors.

Submit your manuscript here: http://www.dovepress.com/international-journal-of-nanomedicine-journal 